\title{
Influence of Overweight and Obesity on Pelvic Tilt, Q-Angle and Foot Posture in Both Genders
}

\author{
MAZHAR ALI BHUTTO ${ }^{1}$, AZADEH SHADMAHER ${ }^{2}$, M. RAZA HADIAN ${ }^{3}$, SAEED TALEBIAN $^{4}$, SYED ARSLAN ASAD ${ }^{5}$ \\ ${ }^{1} P T$, PhD Candidate, Physical Therapy Department, School of Rehabilitation, Tehran University of Medical Sciences, Tehran, Iran \\ 2,3,4 PT, PhD, Physical Therapy Department, School of Rehabilitation, Tehran University of Medical Sciences, Tehran, Iran \\ ${ }^{5}$ PT, PhD, Physical Therapy Department, School of Rehabilitation, University of Lahore, Pakistan \\ Corresponding author: Azadeh Shadmehr, School of Rehabilitation, Piche Shemiran, Enghelab Street, Tehran, PO Box 1148965141, \\ Contact: +982177528468, Email: shadmehr@tums.ac.ir
}

\begin{abstract}
Background: Normal movement pattern and its relationship with various segments of the body create a kinetic chain for performing certain activities. The abnormal patterns of movement may precipitate risk of injuries at any segmentleg from the pelvis to the foot [1-3]. Obesity has been marked as risk factor for postural instability. The mechanism of altering body geometry due to excessive deposition of body fat at certain areas like abdomen and hips increases the stress on the musculoskeletal system leading to postural-instability.

Aim: In study the effects of obesity on different segments of lower limb are being focused.

Place \& Duration: Data was collected in Pakistan. Study duration April 2020 to April 2021

Methods: After ethical approval, Consent and explanation of procedure the population was subdivided into three groups on the bases of BMI, with 50 samples in each group.

For Pelvic tilt and Q-angle measurement the image captured with 13-megapixel camera and angle was measured[4]in computer with the help of Microdicom software. To evaluate foot posture, the foot posture index tool was used. The ICC95\% calculated for validity and reliability of photography.

Results: Validity and reliability test ICC 95\% confidence interval for pelvic tilt and Q-angle measurement with photography method was $0.991(0.974-.997)$ and $0.949(0.878-.981)$, with P-value 0.0001 . There is significant different in pelvic tilt, $\mathrm{Q}$-angle and foot posture of subjects with normal, overweight and obesity.

Conclusion: Obesity has significant effect on Q-angle and pelvic tilt and foot posture.

Key words: Q-angle, Posture, Pelvic, Obesity, Influence.
\end{abstract}

\section{INTRODUCTION}

Normal movement pattern and its relationship with various segments of the body create a kinetic chain for performing certain activities. Studies suggest that abnormal patterns of movement precipitate risk of injuries in lower limb which may occur at any segment of the lower limb from the pelvis to the foot.[1-3]There is an assumption that abnormal gait pattern may lead to patellofemoral pain syndrome, which may be due to flattening, inversion or eversion of forefoot, pelvic tilt, hip antiversion, antitorsion, knee valgus and varus deformities. Several past studies on flat foot have reported that instability may affect the tibial and femoral internal rotation ultimately effecting the Patellar alignment [5-9]. Deviations in morphology of human foot which may be planus, also known as flat foot, increased arch height of foot (cavus) and rectus or regular foot are clinically assessed with radiology, foot posture index (FPI) and footprints method. Foot prints method is less reliable Schwartz et al. whereas radiology is an expensive method, therefore FPI was developed in which diagnosis is possible with direct evaluation through visual and palpitation of surface land marks [7]and has remained method of choice in many researches[8, 9].

Obesity has been marked as risk factor for posturalinstability during performing activities. The mechanism of altering body geometry due to excessive deposition of body fat at certain areas like abdomen hips and trunk creates imbalance in body's balance performance which ultimately initiates stress on the musculoskeletal system leading to instability. Vestibular, visual and proprioceptive sense is responsible to produce stability in human musculoskeletal system,deficits among any of them may leads to postural instability[10]. In obesity, increased pressure due to heavy mass in any part of the body,usually decreases proprioceptive sense [11] and increased fat mass at abdominal area causes shifting of the line of gravity anteriorly due to imbalance in weight distribution because of more increased weight on anterior part of trunk. Thus unequal transfer of body weight results in unnecessary muscular work and ultimately fatigue making one more susceptible to injuries.

Pelvic tilt is a result of muscles imbalance or is secondary to increased lumber lordosis, usually initiates pain in lower back and some time in hip region as well[12].Correlations of obesity with postural instability have been reported in many previous works[11].Lumber spine lordosis due to excessive body weight at abdominal areaeventually leads to anterior pelvic tilt, normal recommended range of pelvic tilt is between 4.9 to 11 degrees posterior to anterior $[13,14]$ and has been measured with radiological image and digital camera photography in past.Quadriceps muscle pull direction may alter the tibial rotation by increasing or decreasing angle of pull.This ultimately may affect the foot alignment and may lead to foot posture deformities. In current study it is believed that altered direction of weight transfer from upper trunk to lower limb, due to obesity is responsible for increasing Q-angle which in turn createspelvic tilt knee and foot problems. Therefore, this study aims to find out the correlation of Q-angle with pelvic tilt and foot posture of both genders in different BMls. 


\section{METHODS}

The approval was taken from the ethical committee of Tehran University of Medical Science (TUMS) Iran and University of Lahore Pakistan. This study was designed and carried out at physiotherapy department TUMS and data was collected from population of Pakistan, with simple random sampling technique. Consent forms were signed by participants after receiving explanation about all purposes and procedures of this study. Age, height and weight of participants were taken to calculate BMI, with normal weight range between 18.5 to $24.9 \mathrm{~kg} / \mathrm{m}^{2}$, over weight range between 25.0 to $29.9: \mathrm{kg} / \mathrm{m}^{2}$ and obese range 30.0 $\mathrm{kg} / \mathrm{m}^{2}$ and above. Sample size of 150 limbs was divided into three groups on the basis of their calculated BMI values into normal weight, overweight and obese groups. Each group had 50 limbs, with 25 legs in right and left side each.

Inclusion criteria: Subjects were shortlisted by following age between 20 to 45 yearsin both genders and BMl of normal weight, overweight and obese.

Exclusion criteria: Subjects with history of trauma, symptomatic knee, neurological disorder, deformities either genetically or acquired and amputationare not considered in this study.

Procedures: For measurement of Pelvic tilt and Qangle,digital camera of mobile OPPO F1s with capacity of 13-megapixel, specifications of ( $f / 2.2)$ focal length aperture was used to capture images and desired angles were calculated by using the Microdicom software in computer. To evaluate foot inversion, eversion and normal foot, the foot posture index tool was used.

Camera was placed on a tripod stand at a height of $90 \mathrm{~cm}$ and at a distance of $290 \mathrm{~cm}$ from the subject in a nonreflective environment[4]. Subjects for uniformity in all samples were standing on a marked floor with indicators for foot placement at $7 \mathrm{~cm}$ distance between heels and 10 degrees of forefoot external rotation. Hands were folded over the chest.Body surface landmarks were palpated and marked with reflective markers.

Pelvic tilt angle is assessed by using horizontal plane line between Anterior Superior iliac spines(ASIS) to posterior Superior iliac spine (PSIS) on a horizontal plane. Angle between horizontal plane and line from PSIS to ASIS near junction of two lines[4] was calculated with the help of Microdicom software in computer. Images were taken in lateral view Figure 1.

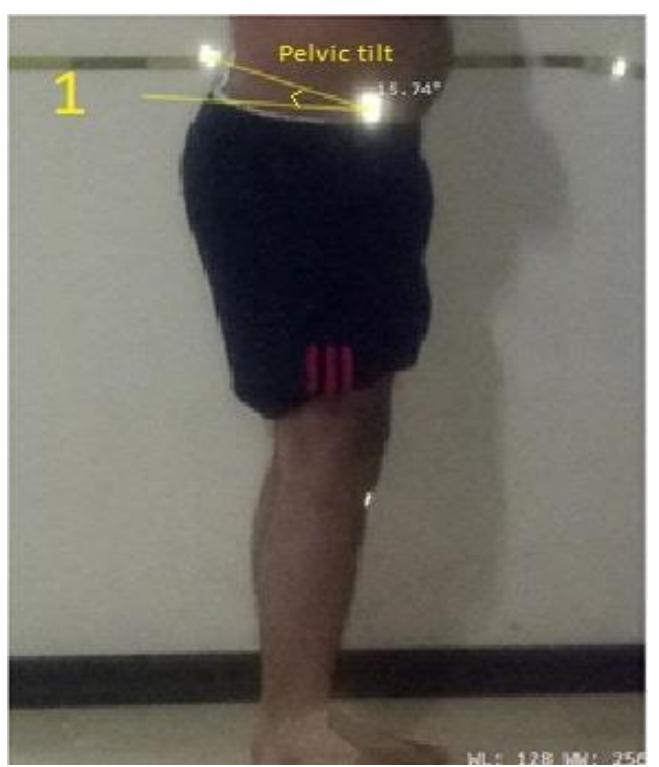

Figure1: Showinglateral view image with Markers on ASIS, PSIS andPelvic tilt angle measurement on Microdicon Software.

For Q-angle, Images of lower limbswere taken in anterior view with a digital camera and the photo was used for calculation of $Q$-angle by drawing a line from Anterior Superior iliac spines (ASIS) to the center of patella (COP), another line was drawn from the tibial tuberosity (TT) marker to the COP and upwards angle calculated between these two lines by using the Microdicom software Figure 2 and 3 . The center of patella was located with the help of Vernier caliper.

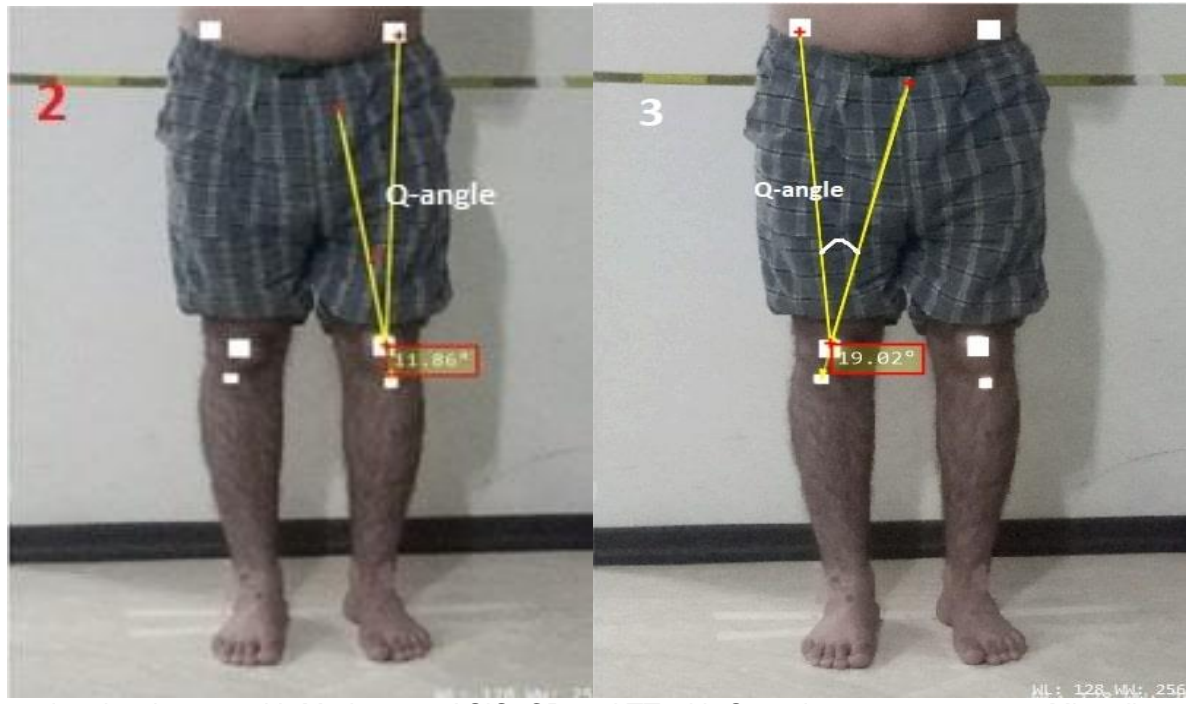

Figure2\&3: showing anterior view image, with Markers on ASIS, CP and TT with Q-angle measurement on Microdicom software. 
For Foot posture the three dimensional evaluation of foot in standing position with relaxed foot were performed followed by assessment of anterior, middle and posterior part of the foot (fore, mid and hind foot) with six steps (1) Palpation of talar head (2) Supra / infra lateral malleolus arch (3) the calcaneus inversion and eversion of (4) talonavicular joint prominence (5) Medial longitudinal arch height (6) abduction and adduction of the forefoot.The summation of FPI score is suggested to diagnose as normal foot, supinated and pronated. The scores were allocated such that if the above mentioned landmarks were slightly palpable a score of \pm 1 was given and if not palpable a score \pm 2 was given. Negative sign (-) indicates inversionand positive sign (+) indicates eversion of the foot. Total score of FPI ranges from -12 (maximum supinated) to 12 (maximumpronated).

Statically Analysis: The data was analyzed by using SPSS version 22. The Kolmogorov Smirnov test was applied to determine the normal distribution of data. The intergroup difference significance in pelvic tilt, $Q$-angle, foot posture and BMI was determined by using oneway ANOVA test. Inter group difference calculated with Post hoc test.Pvalue $<0.05$ was accepted as significant. Validity and reliability of Digital photography in measuring Q-angle and pelvic tilt were measured with ICC 95\%[4].

\section{RESULTS}

The reliability test with ICC 95\% confidence interval for pelvic measurement with Digital photography method was 0.991 (0.974-.997) and P-value 0.0001. This is highly reliable, valid and significant. Reliability in Q-angle measurement by using Digital photography and Microdicom software was ICC 0.949(0.878-.981) highly reliable and a significant P-value of 0.0001 was noted. Q-angle in both genders is significant different with $P$-value of 0.0001 . Female reported minimum 12 degree and maximum 21 degrees $Q$ angle, whereas males were with 10 minimum to 20 degree maximum $\mathrm{Q}$-angle. Bilateral Asymmetry in males was reported 0.3 degree and 0.1 degrees in females.

In results of one way ANOVA tests there is significant differencein Pelvic Tilt of normal, overweightand obese weights subjects (table1). ThePost Hoc test results for Pelvic tilt measurement is significantly different among normal weight and Overweight subjects. There is also same significant differencein normal weight and obese subjects pelvic tilt angle(table2).There is significant difference between $\mathrm{Q}$-angle in normal weight, overweight and obese weights subjects on using one way ANOVA tests (table3). Q-angle Bonferroni Post Hoc test results showed there is significant difference in normal weight, overweight and obese groups with each other (table 4).

There is significant difference between foot posture in normal weight, overweight and obese weights subjects groupson right and left side (table 5).

Table 1 In One way ANOVA tests the Mean (SD) and 95\% confidence interval of pelvic tilt (degrees) in subjects with different BMls. (Total $\mathrm{n}=150$ )

\begin{tabular}{|l|l|l|l|l|}
\hline BMI & Pelvic tilt side & Mean(SD) & $95 \%$ confidence interval & p-value in BMI Groups \\
\hline \multirow{2}{*}{ Normal weight } & Right & $9.8(3.6)$ & $(8.3,11.3)$ & \\
\cline { 2 - 4 } & Left & $10.5(2.9)$ & $(9.3,11.7)$ & \multirow{2}{*}{0.0000} \\
\cline { 2 - 4 } Overweight & Right & $14.3(1.7)$ & $(13.6,15.0)$ & \\
\cline { 2 - 4 } & Left & $13.8(1.9)$ & $(14.9,15.9)$ & \\
\cline { 2 - 4 } & Right & $15.5(1.3)$ & $(13.9,15.9)$ & \\
\hline
\end{tabular}

Table 2 Bonferroni test results for Pelvic tilt in normal weight, overweight and obese population

\begin{tabular}{|c|c|c|c|c|c|}
\hline Side & Weight & Groups & Mean Difference & Std. Error & P-value \\
\hline \multirow{4}{*}{ Right } & \multirow{2}{*}{ over weight } & Obese & -1.12000 & .69213 & 0.330 \\
\hline & & normal weight & 4.50080 & .69213 & 0.000 \\
\hline & \multirow{2}{*}{ Obese } & overweight & 1.12000 & .69213 & .330 \\
\hline & & Norma Iweight & 5.62080 & .69213 & 0.000 \\
\hline \multirow{4}{*}{ Left } & \multirow{2}{*}{ over weight } & Obese & -.87120 & .64641 & 0.546 \\
\hline & & normal weight & 3.24080 & .64641 & 0.000 \\
\hline & \multirow[b]{2}{*}{ Obese } & over weight & .87120 & .64641 & 0.546 \\
\hline & & normal weight & 4.11200 & .64641 & 0.000 \\
\hline
\end{tabular}

The mean difference is significant at the .05 level.

Table 3, In One way ANOVA tests the Mean (SD) and 95\% confidence interval of Q-angle(degrees) in subjects with different BMls. Using one way ANOVA test

\begin{tabular}{|l|l|l|l|l|}
\hline BMI & Q-angle in leg side & Mean(SD) & $95 \%$ confidence interval & $p$-value \\
\hline \multirow{2}{*}{ Normal weight } & Right & $12.95(1.65)$ & $(12.27,13.63)$ & \\
\cline { 2 - 4 } & Left & $12.94(1.66)$ & $(12.2,13.6)$ & \multirow{2}{*}{0.0000} \\
Over Weight & Right & $15.94(1.28)$ & $(15.4,16.4)$ & \\
\cline { 2 - 4 } & Left & $15.7(1.16)$ & $(15.2,16.2)$ & \\
\cline { 2 - 4 } Obese & Right & $18.42(1.24)$ & $(17.9,18.9)$ & $(17.5,18.6)$ \\
\hline
\end{tabular}


Influence of Overweight and Obesity on Pelvic Tilt, Q-Angle and Foot Posture in Both Genders

Table 4 Bonferroni test results for Q-angle in normal weight, overweight and obese population

\begin{tabular}{|c|c|c|c|c|c|c|}
\hline Side & Dependent Variable & weight & Groups & Mean Difference & Std. Error & $\mathrm{P}$-value \\
\hline \multirow{4}{*}{ Right } & \multirow{4}{*}{ Q-angle } & \multirow{2}{*}{ Over weight } & Obese & -2.48800 & .39779 & 0.000 \\
\hline & & & normal weight & 2.98800 & .39779 & 0.000 \\
\hline & & \multirow[b]{2}{*}{ Obese } & over weight & 2.48800 & .39779 & 0.000 \\
\hline & & & normal weight & 5.47600 & .39779 & 0.000 \\
\hline \multirow{4}{*}{ Left } & \multirow{4}{*}{ Q-angle } & \multirow{2}{*}{ over weight } & Obese & -2.41640 & 39131 & 0.000 \\
\hline & & & normal weight & 2.78000 & .39131 & 0.000 \\
\hline & & \multirow[b]{2}{*}{ Obese } & over weight & 2.41640 & .39131 & 0.000 \\
\hline & & & Normal weight & 5.19640 & .39131 & 0.000 \\
\hline
\end{tabular}

The mean difference is significant at the .05 level.

Table 5: ANOVA Foot postures (Normal, Inverted and Everted) in subjects with different BMls.

\begin{tabular}{|l|l|l|l|l|l|l|l|}
\hline \multirow{2}{*}{ FPI } & Right Side & Left Side & normal weight & p-value \\
\cline { 2 - 8 } & over weight & Obese & normal weight & over weight & Obese & \multirow{2}{*}{0.0000} \\
\hline Normal & $18(72.0)$ & $14(56.0)$ & $21(84.0)$ & $18(72.0)$ & $14(56.0)$ & $21(84.0)$ & \\
\hline Inverted & $1(4.0)$ & $0(0.0)$ & $2(8.0)$ & $1(4.0)$ & $0(0.0)$ & $2(8.0)$ & \\
\hline Everted & $6(24.0)$ & $11(44.0)$ & $2(8.0)$ & $6(24.0)$ & $11(44.0)$ & $2(8.0)$ & \\
\hline Total & 25 & 25 & 25 & 25 & 25 & 25 & \\
\hline
\end{tabular}

\section{DISCUSSION}

Digital Photography method and use of Microdicom software remained method of choice in measuring different body angle. The method used in this study for measurement of $\mathrm{Q}$-angle is relatively less expensive and do not have health hazards compared to previously, Qangle was measured with radiology and goniometer[15, 16]. The method used in this study is relatively easier to perform, requires minimal expertise and has good validityreliability.

The presence of increased spinal lordosis and scoliosis were noted in subjects with pelvic tilt and anteversion of hip joint by Gurney and legaye et al[17, 18]. This may cause the increased load on spinal facets joints, ultimately leading to back complications[19, 20]. Another studyresembling this article assumed that Q-angle and foot posture may be affectedby lordosis or obesity which in turn alters the pelvic morphology. Similarly any deviation in the foot posture and Q-angle itself may also lead to alteration in pelvic position.

Increased Q-angle has been considered as a risk factor for patellar subluxation and Chondromalacia patella. It has different mathematical values for normal and increased range given by several authors in past studies with various assumptions. One such example is the wider pelvic size in female gender being responsible for $\mathrm{Q}$-angle variation[21].Another study comparedthe length of femur bone to the width of pelvis[22], but the above assumptions were rejected by Byl, T. and J.A. Cole[23]. Thereforethis study assumed thatbody mass index mayhave an influence on $Q$-angle, and the results obtained depict significant $p$ value of 0.0001 .

Q-angle Mean (SD) values in Obesewere reported to be 18.42 (1.24), Overweight- 15.94 (1.28) and Normal weight - 12.95 (1.65) which bear a resemblance with most of the past findings[16, 24]but they were documented as normal Q-angle and increased Q-angle. This study suggests that obesity may have an effect on $Q$-angle and beingoverweight might be the cause for an increased Qangle upwards of 15 degrees where a Q-angle of 16 degrees and above is considered abnormally high Qangle.
Bilateral Asymmetry in left and right in both gender were not reported with mark-able difference, ranging between mean and SD15.3 \pm 2.6 to $15.0 \pm 2.6$ in male right and left side with difference of 0.3 degree and16.5 \pm 2.6 to16.6 \pm 2.1 in right and left side of female with almost no asymmetric difference. The result were almost same in Jaiyesimi, $A$. and $O$. Jegede work, who reported female Right QA 16.93 and Left QA 16.30 with a difference of 0.35 degree.

Pelvic tilt in normal weight subjects Mean (SD) 9.8 (3.6) was noted, Overweight Mean (SD) 14.3 (1.7) and in obese pelvic tilt Mean (SD) was 15.5 (1.3), which illustrates that pelvic tilt has acorrelation with being Overweight and obese. Previous studies have already proven that due to segmental inter-connection, lumber lordosis hasan effect on pelvic tilt.The results of this study are in favor of thesame assumption, with an addition that obesity is also responsible for pelvic tilt. More studies are recommended on the effects of obesity on lumber spine and pelvic tilt for further clarity.

While working on foot posture and pelvic tilt, Khamisand Yizhar induced foot hyper pronation in standing postureand found pelvis to be anteriorly tilted and thigh internally rotated[25].The results of the current study showed that $44 \%$ of obese individuals were found to haveEverted foot and $90 \%$ reported anterior pelvic tilt which shows possible correlation between obesity, pelvic tilt and foot deviation.

Acknowledgement: This study is part of Ph.D. thesis and granted by Tehran University of Medical Sciences, Tehran, Iran (grant 99-3-163-51116). The Authors would like to thank the Head of Physiotherapy Department, School of Rehabilitation, Tehran University of Medical Sciences for their support and cooperation and all the participants for their contribution to this study. We wish to report no conflict of interest and declare that we are solely responsible for the content and writing of the manuscript.

Limitation: The limitations of this study were difficulty in finding voluntary participant especially in females. Difficulty in palpitation of bony land marks in obese population. 


\section{CONCLUSION}

Body Mass Index has significant effect on pelvic tilt, Qangle and foot posture. Obesity has significant effect onQangle and pelvic anterior tilt. Although number of cases of foot eversion in obesity are not prominent, but being obese in the long term may lead to further changes in foot posture. Further research is recommended with larger sample size and equivalent participant representing both genders

\section{REFERENCES}

1. Williams, D.S., et al., Lower extremity kinematic and kinetic differences in runners with high and low arches. Journal of Applied Biomechanics, 2001. 17: p. 153-163.

2. Williams, D.S. and I. McClay, Measurements used to characterize the foot and the medial longitudinal arch: reliability and validity. Physical therapy, 2000. 80 9: p. 86471.

3. Park, S.-K. and D.J. Stefanyshyn, Greater $Q$ angle may not be a risk factor of patellofemoral pain syndrome. Clinical biomechanics, 2011. 26(4): p. 392-396.

4. Bonifácio, D., et al., Intra-and inter-rater reliability in photogrammetric pelvic tilt angles analysis. 2013, MA Healthcare London.

5. Duffey, M.J., et al., Etiologic factors associated with anterior knee pain in distance runners. Medicine and science in sports and exercise, 2000. 32(11): p. 1825-1832.

6. Messier, S.P., et al., Etiologic factors associated with patellofemoral pain in runners. Medicine and science in sports and exercise, 1991. 23(9): p. 1008-1015.

7. Buchbinder, M., N. Napora, and E. Biggs, The relationship of abnormal pronation to chondromalacia of the patella in distance runners. Journal of the American Podiatric Medical Association, 1979. 69(2): p. 159-162.

8. Callaghan, M. and V. Baltzopoulos, Gait analysis in patients with anterior knee pain. Clinical Biomechanics, 1994. 9(2): p. 79-84.

9. Powers, C.M., R. Maffucci, and S. Hampton, Rearfoot posture in subjects with patellofemoral pain. Journal of Orthopaedic \& Sports Physical Therapy, 1995. 22(4): p. 155160.

10. Loram, I.D. and M. Lakie, Direct measurement of human ankle stiffness during quiet standing: the intrinsic mechanical stiffness is insufficient for stability. The journal of physiology, 2002. 545(3): p. 1041-1053.
11. Son, S.M., Influence of obesity on postural stability in young adults. Osong public health and research perspectives, 2016. 7(6): p. 378-381.

12. Levine, D. and M.W. Whittle, The effects of pelvic movement on lumbar lordosis in the standing position. Journal of Orthopaedic \& Sports Physical Therapy, 1996. 24(3): p. 130135.

13. De Souza, S.A.F., et al., Postural changes in morbidly obese patients. Obesity surgery, 2005. 15(7): p. 1013-1016.

14. Rodacki, A.L.F., et al., Body mass as a factor in stature change. Clinical Biomechanics, 2005. 20(8): p. 799-805.

15. Livingston, L.A. and S.J. Spaulding, OPTOTRAK Measurement of the Quadriceps angle using standardized foot positions. Journal of athletic training, 2002. 37(3): p. 252.

16. Woodland, L.H. and R.S. Francis, Parameters and comparisons of the quadriceps angle of college-aged men and women in the supine and standing positions. The American journal of sports medicine, 1992. 20(2): p. 208211.

17. Gurney, B., Leg length discrepancy. Gait \& posture, 2002. 15(2): p. 195-206.

18. Legaye, J., et al., Pelvic incidence: a fundamental pelvic parameter for three-dimensional regulation of spinal sagittal curves. European Spine Journal, 1998. 7(2): p. 99-103.

19. Shirazi-Adl, A. and G. Drouin, Load-bearing role of facets in a lumbar segment under sagittal plane loadings. Journal of biomechanics, 1987. 20(6): p. 601-613.

20. Steinberg, E., et al., $A$ comparative roentgenographic analysis of the lumbar spine in male army recruits with and without lower back pain. Clinical radiology, 2003. 58(12): p. 985-989.

21. Outerbridge, R., Further studies on the etiology of chondromalacia patellae. The Journal of bone and joint surgery. British volume, 1964. 46(2): p. 179-190.

22. Grelsamer, R., A. Dubey, and C. Weinstein, Men and women have similar $Q$ angles: a clinical and trigonometric evaluation. The Journal of bone and joint surgery. British volume, 2005. 87(11): p. 1498-1501.

23. Byl, T., J.A. Cole, and L.A. Livingston, What determines the magnitude of the $Q$ angle? A preliminary study of selected skeletal and muscular measures. Journal of Sport Rehabilitation, 2000. 9(1): p. 26-34.

24. Mo, J., Manual of Orthopedic Surgery. Park Ridge, IL: American Orthopaedic Association, 1972.

25. Khamis, S., et al., The relationship between foot and pelvic alignment while standing. Journal of human kinetics, 2015. 46: p. 85. 